\title{
INFLUENCE OF THE LAMINAR BURNING VELOCITY ON THE TRANSFER FUNCTIONS OF PREMIXED METHANE AND PROPANE SWIRL FLAMES
}

\author{
F. Di Sabatino; T.F. Guiberti \\ Clean Combustion Research Center \\ King Abdullah University of Science and Techonolgy \\ Thuwal, 23955-6900, Saudi Arabia
}

\author{
J.P. Moeck \\ Norwegian University of Science and Technology \\ Department of Energy and Process Engineering \\ 7491 Trondheim, Norway
}

\author{
W.L. Roberts, D.A. Lacoste \\ Clean Combustion Research Center \\ King Abdullah University of Science and Techonolgy \\ Thuwal, 23955-6900, Saudi Arabia
}

\begin{abstract}
The effects of the laminar burning velocity $\left(S_{L}\right)$, on the transfer functions of propane-air and methane-air swirl flames is experimentally investigated. Five equivalence ratios for each fuel are selected, to yield different values of $S_{L}$. The flame transfer function (FTF), is obtained by comparing the velocity fluctuations of the incoming flow, measured with a hot wire, to the heat release rate oscillations, collecting $\mathrm{OH}^{*}$ chemiluminescence with a photomultiplier tube. Phase-locked images of $\mathrm{OH}^{*}$ chemiluminescence are also acquired to analyze the flame dynamics during the forcing cycle. The unforced velocity fields are measured by particle image velocimetry to assess the effects of $S_{L}$ on the flow fields. Changing the laminar burning velocity affects mainly the gain around $176 \mathrm{~Hz}$ and $336 \mathrm{~Hz}$. This paper focuses on $336 \mathrm{~Hz}$. The flame vortex roll-up is recognized as a key parameter controlling the gain of the FTF around $336 \mathrm{~Hz}$. The analysis highlights that $S_{L}$ influences the gain response around $336 \mathrm{~Hz}$ in two competing ways: first, it enhances the flame vortex roll-up and second, it affects the stabilization distance of the flame, which influences the vortex generated by acoustic forcing.
\end{abstract} \footnotetext{
*Address all correspondence to this author at: francesco.disabatino@kaust.edu.sa.

\section{INTRODUCTION}

Thermoacoustic instabilities are a major problem for gas turbines operating in premixed lean conditions. These instabilities result from the coupling between the unsteady heat release from the flame and the acoustic modes of the combustor. This interaction can be very detrimental for the gas turbine and it can even lead to structural failure of the system $[1,2]$. Therefore, it is important to understand the response of the flame to acoustic perturbations. This is often done using the flame transfer function (FTF) formalism. For premixed flames, the FTF relates the heat release rate fluctuations from the flame to the velocity oscillations of the incoming flow [3,4]. Since it is very expensive to perform simulations or experiments for every operating condition of a gas turbine, simple relations with parameters that are easy to measure need to be defined, in order to predict the FTF at a given operative point. Flame base angle and flame length are examples of parameters that have been used in previous studies to predict FTF $[5,6]$. Therefore, analyzing how different parameters affect the FTF is of interest.

The dynamics of premixed swirl flames subjected to acoustic forcing is qualitatively well understood. The interaction between the fluctuations of the swirl number and the flame vortex

$$
\text { Copyright (c) } 2019 \text { by ASME }
$$


roll-up controls the response of the flame [4,6-8]. Typically, the FTF gain features a local minimum followed by a local maximum. At the frequency $f_{l}$ for which swirl number fluctuations are largest, the FTF gain is locally minimal. At frequency $f_{2}$ for which the flame vortex roll-up is the most intense, the FTF gain shows a maximum. For some configurations, the gain of the FTF shows an additional local maximum at a frequency of around two times $f_{l}$. The phase of the FTF is generally linearly proportional to the forcing frequency $[4,8]$. It is interesting to investigate the parameters controlling the flame vortex roll-up (FVR) mechanism since it has been recognized as a fundamental phenomenon controlling the flame motion at the frequency at which the gain of the FTF shows its local maximum [4, 8, 9]. There have been previous studies that dealt with the investigation of the effects of parameters, such as equivalence ratio $[10,11]$ and pressure [12-14], on the flame response. Bellows et al. [10] showed that the flame response to acoustic forcing, in terms of $\mathrm{CH}^{*}$ emissions from the flame, increases when the equivalence ratio, $\phi$, is increased. They also pointed out that the forcing amplitude at which the FTF gain saturates also increases with $\phi$. Similarly, Kim et al. [11] showed that increasing the equivalence ratio at a fixed forcing frequency yields an increase of FTF gain. They explained that this trend could be generalized in terms of the ratio of the relative length of the flame to convection length scale of the velocity perturbation. Even if these studies gave a useful insight into the effects of such parameters on the flame response, they did not fully investigate their effects on the FVR mechanism.

On the other hand, several studies analyzed the interaction between the flame and the acoustically generated vortex that leads to the FVR $[8,15]$. Bunce et al. $[8]$ discussed the interaction between the fluctuations of the base of the flame and the acoustically generated vortex (AGV). They suggested that, when the flame oscillates close to the shear layer where the AGV is shed, the temperature rise in that region weakens the acoustically generated vortex, and consequently the FVR. Oberleithner et al. [15] analyzed the stability of the shear layer where the AGV is shed for different values of forcing amplitude. They observed that the receptivity of the shear layer defines the size of the acoustically generated vortex and consequently the response of the flame to acoustic forcing. However, these two studies did not analyze the effects of parameters such as $S_{L}$ on the FVR phenomenon. More recently, Gatti etal. [16,17] investigated the influence of swirl intensity and central bluff body on the FTF. They observed that the strength of the AGV defines the response of the flame. This strength is influenced by the forcing frequency, the swirl intensity, and the presence of the central bluff body. Since they performed the characterization of the AGV in cold flow configuration only (without the flame), no information about the interaction between flame parameters, such as $S_{L}$, and the acoustically generated vortex are available. Finally, Gaudron et al. [18] identified several parameters, like Lewis number, flame thick- ness, and laminar burning velocity, that control the response of premixed laminar flames to acoustic forcing. Similarly, it would be interesting to asses the effect of flame properties, such as $S_{L}$, on the transfer function of premixed swirl flames. This is the purpose of the present study.

The main objective is to investigate the influence of the laminar burning velocity on the response of swirl flames to acoustic forcing. Precisely, the influence of $S_{L}$ on the FVR mechanism and consequently on the gain of the FTF is analyzed at the forcing frequency of $336 \mathrm{~Hz}$, which corresponds to a local maximum of the gain.

\section{EXPERIMENTAL SETUP AND PROCEDURE}

In this section, the experimental setup and procedure, as well as the diagnostics used for the analysis of the flame dynamics and the velocity fields, are presented.

\section{Swirl-stabilized burner}

A schematic of the burner is presented in Fig. 1, while its detailed description can be found in [9, 19]. The flow of air and fuel are controlled by thermal mass flow meters (Brooks SLA 58 Series), and mixed 2 meters (more than 200 diameters), upstream of a $120 \mathrm{~mm}$ long plenum to ensure a perfect mixing. After being injected in the plenum, the combustible mixture flows through a honeycomb and a perforated plate to break up the large turbulent structures. Then, the mixture flows through a radial swirler that features a measured swirl number of 0.39 [20,21]. Afterward, the mixture is injected in the combustion section of the burner through an 18-mm injector tube that includes a central rod of $2.5 \mathrm{~mm}$ diameter. The combustion section of the burner is confined by a 100-mm long quartz tube with an inner diameter of $70 \mathrm{~mm}$.

A $900 \mathrm{~W}$ loudspeaker (Beyma 10LW30/N), installed at the bottom of the burner and connected to a high fidelity amplifier (QSC GX5), generates the acoustic forcing needed for this study. The frequency and the amplitude of the forcing signal are controlled by a signal generator (NF WF1973), connected to the amplifier.

\section{Experimental conditions}

Five equivalence ratios for two fuels, methane and propane, are considered in this study. The equivalence ratio, thermal power $\left(P_{t h}\right)$, average bulk velocity $\left(\bar{V}_{b u l k}\right)$, Reynolds number $(\mathrm{Re})$, laminar burning velocity, and adiabatic flame temperature $\left(T_{a d}\right)$, of these ten flames are summarized in Table 1. Direct visualization of the corresponding flames is shown in Fig. 2. 


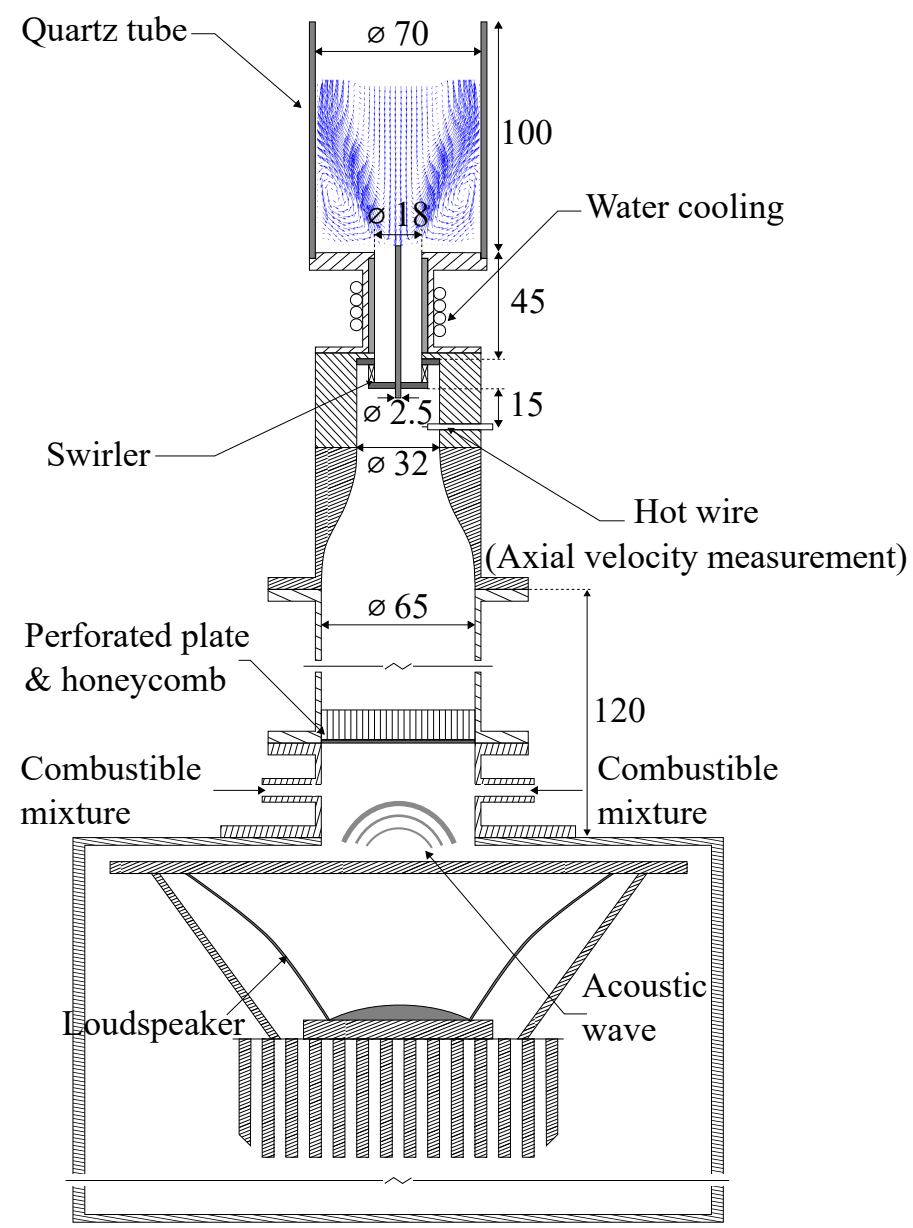

FIGURE 1. SCHEMATIC OF THE BURNER. ALL DIMENSIONS ARE IN MILLIMETERS.

TABLE 1. SUMMARY OF THE EXPERIMENTAL CONDITIONS.

\begin{tabular}{ccccccc} 
Fuel & $\phi$ & $\begin{array}{c}P_{\text {th }} \\
(\mathrm{kW})\end{array}$ & $\begin{array}{c}\bar{V}_{\text {bulk }} \\
(\mathrm{m} / \mathrm{s})\end{array}$ & $\mathrm{Re}$ & $\begin{array}{c}S_{L} \\
(\mathrm{~m} / \mathrm{s})\end{array}$ & $\begin{array}{c}T_{a d} \\
(\mathrm{~K})\end{array}$ \\
\hline $\mathrm{C}_{3} \mathrm{H}_{8}$ & 0.69 & 4.4 & 6.5 & 8,000 & 0.20 & 1863 \\
& 0.70 & 4.4 & 6.5 & 8,000 & 0.22 & 1880 \\
& 0.74 & 4.7 & 6.5 & 8,000 & 0.24 & 1947 \\
& 0.79 & 4.7 & 6.3 & 7,600 & 0.30 & 2027 \\
& 0.83 & 5.0 & 6.3 & 7,600 & 0.32 & 2087 \\
& & & & & & \\
$\mathrm{CH}_{4}$ & 0.65 & 3.8 & 6.4 & 7,400 & 0.15 & 1754 \\
& 0.67 & 3.9 & 6.4 & 7,400 & 0.16 & 1788 \\
& 0.70 & 4.0 & 6.4 & 7,400 & 0.19 & 1839 \\
& 0.73 & 4.0 & 6.2 & 7,100 & 0.20 & 1888 \\
& 0.76 & 4.2 & 6.2 & 7,100 & 0.25 & 1935 \\
\hline
\end{tabular}

The values of the laminar burning velocity are evaluated using Cantera [22] for a freely propagating flame with an initial temperature of $300 \mathrm{~K}$ using the USC-II mechanism [23] and a mixture-averaged mass diffusion model. Then, the calculated values of $S_{L}$ from Cantera are averaged with the values extrapolated from [24-29]. Since the propane flames feature a Lewis number different from unity $(\mathrm{Le}=1.89)$, the unstretched laminar burning velocity, presented in Table 1 , could be affected by the local curvature of the flame. However, it has been shown in $[30,31]$ that for the range of forcing frequencies considered in this work, the non-unity Lewis number effects on the laminar burning velocity are negligible. Therefore, differential-diffusion effects are ignored for the remaining analysis and the local burning velocity will be considered as equal to the unstretched laminar burning velocity $S_{L}$, presented in Table 1 .

To have a meaningful comparison between the ten flames, the experimental conditions are chosen to exhibit the local extrema of the gain of the FTF around the same frequencies. This is obtained by adjusting the average bulk velocity of the flows.

\section{Diagnostics}

The fluctuations of the global heat release rate from the flame and the flow velocity oscillations need to be measured to determine the FTF. The heat release rate fluctuations are determined from the global $\mathrm{OH}^{*}$ chemiluminescence from the flame by using a photomultiplier tube (Hamamatsu H10721), equipped with a $10 \mathrm{~nm}$ bandpass filter centered at $310 \mathrm{~nm}$ (ZBPA310 ASAHI Spectra Co.). This technique can be used since only perfectly premixed flames are considered in this study [32,33]. The flow velocity oscillations are measured with a hot wire probe (Dantec miniCTA), placed $7 \mathrm{~cm}$ from the outlet of the injection tube, upstream of the swirler. This does not introduce any significant modifications to the FTF for forcing frequencies lower than $1 \mathrm{kHz}$ [34]. An oscilloscope (Agilent Technologies Infiniium 2.5 $\mathrm{GHz}$ ), is used to record the forcing signal, the photomultiplier (PMT) signal, and the hot wire signal.

An intensified CCD camera (Princeton Instruments PIMAX), equipped with a UV lens (105 mm Coastal Optics), and a $10 \mathrm{~nm}$ bandpass filter centered at $310 \mathrm{~nm}$ (ZBPA310 ASAHI Spectra Co.), collects $\mathrm{OH}^{*}$ chemiluminescence that is used to analyze the flame motion during the forcing cycle. An exposure time of $200 \mu$ s is imposed for all the experimental conditions.

A particle image velocimetry (PIV) system is implemented to investigate the velocity fields. This system is composed of a $10 \mathrm{~Hz}$ dual pulse Nd:YAG laser (Litron Nano L200-15 PIV), and a 1200x1600 pixels dual frame CCD camera (LaVision Imager Pro X), equipped with a visible lens (60 mm AF Micro Nikkor), and a $10 \mathrm{~nm}$ bandpass filter centered at $532 \mathrm{~nm}$ (LaVision VZ170117). Each laser beam features an average energy per pulse of $26 \mathrm{~mJ}$ at $532 \mathrm{~nm}$, and it is converted into a $1 \mathrm{~mm}$ thick and $60 \mathrm{~mm}$ high laser sheet using a series of spherical and cylindrical lenses. 


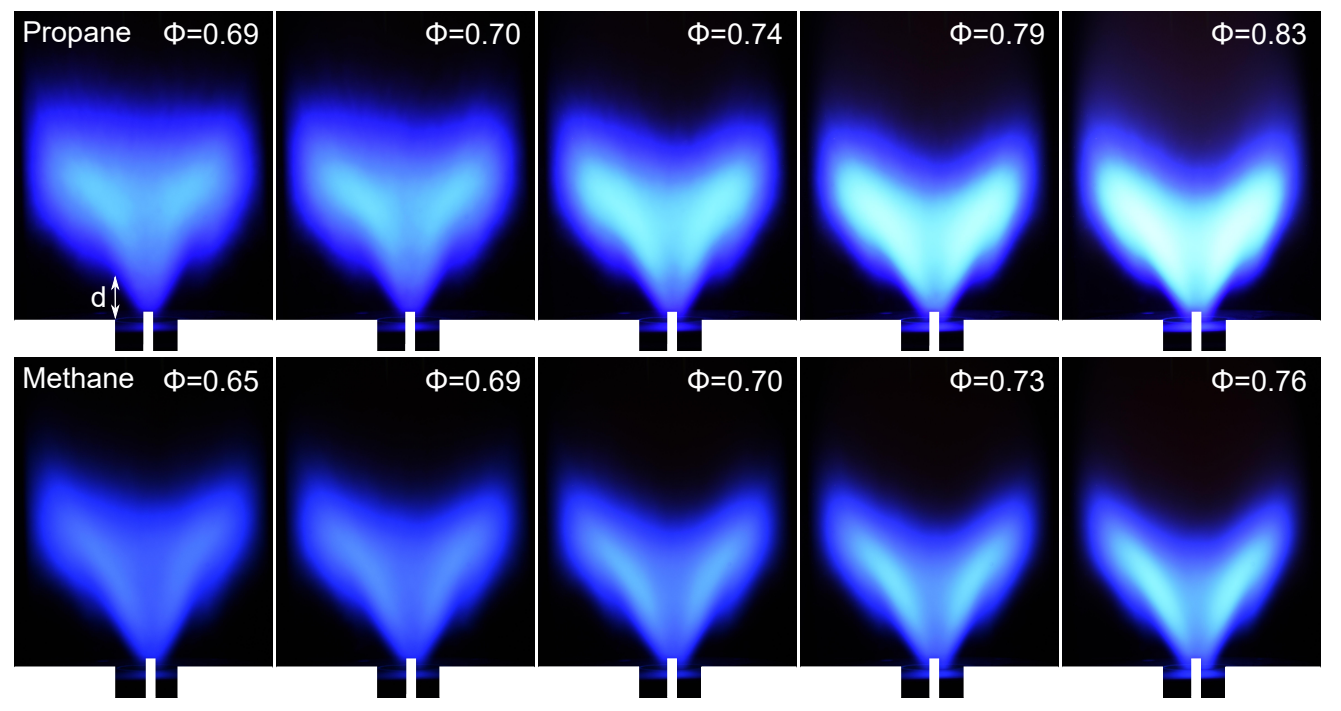

FIGURE 2. DIRECT VISUALIZATION OF THE FLAMES CONSIDERED IN THIS STUDY. THE EXPOSURE TIME IS $1.6 \mathrm{~s}$ FOR ALL THESE IMAGES. THE STABILIZATION DISTANCE (d) BETWEEN THE FLAME AND THE NOZZLE TIP IS HIGHLIGHTED.

The axial and radial components of the velocity fields are measured along the central plane of the axisymmetric burner. The combustible mixture is seeded with titanium dioxide $\left(\mathrm{TiO}_{2}\right)$ particles. A $16 \times 16$ pixels final interrogation area and 50\% overlap is imposed to the software (LaVision DaVis 8.4.0), used to process the data.

\section{Experimental procedure}

For each experimental condition considered in this study, the acoustic forcing is started after the flame is stabilized and the thermal steady state is reached. The frequency of the forcing signal is increased from $32 \mathrm{~Hz}$ to $400 \mathrm{~Hz}$ by incremental steps of $16 \mathrm{~Hz}$. The amplitude of the forcing signal is adjusted in order to maintain a velocity fluctuation equal to $10 \%$ of the bulk velocity, for all frequencies and all the flames considered. This percentage is chosen as a compromise between a linear response of the flame and the natural level of turbulence fluctuations (about 5\%). At each analyzed frequency, the hot wire, photomultiplier tube, and forcing signals are recorded for $10 \mathrm{~s}$.

The flame motion is investigated at the forcing frequency of $336 \mathrm{~Hz}$, that is one of the frequencies at which the gain of the FTF is highly sensitive to the change in laminar burning velocity. The forcing signal is divided into 10 phases with $36^{\circ}$ interval to collect phase-locked averaged images of $\mathrm{OH}^{*}$ chemiluminescence from the flame. The phase equal to zero corresponds to $u^{\prime}=0$ with $\frac{\partial u}{\partial t}>0$. At each phase of the forcing cycle, a total of 1000 images of $\mathrm{OH}^{*}$ chemiluminescence are collected and averaged. Due to the axisymmetric nature of the flame, an Abeldeconvolution technique is also applied.

The average velocity field of the unforced flames is eval- uated averaging 800 instantaneous velocity fields. Then, a 5x5 Gaussian smoothing filter is applied. Afterward, the Qcriterion $[15,35]$ is applied to the field to highlight coherent flow structures such as the ORZ. The Q-criterion is defined as $Q=0.5\left(\Omega^{2}-S^{2}\right)$, where $\Omega$ represents the magnitude of the vorticity tensor and $S$ the shear strain rate. The overall maximum value of the Q-criterion is also used to normalize the Q-criterion fields of each flame analyzed in this study.

\section{RESULTS AND DISCUSSION}

In this section, the definition of the FTF is first introduced, together with the trend of its gain and phase for the propane flames. Then, the behavior of the gain at $336 \mathrm{~Hz}$ as a function of the equivalence ratio and the laminar burning velocity is presented for both fuels. Next, the dynamics of the flames and the unforced velocity fields are analyzed for different values of $S_{L}$. At last, the parameters controlling the gain response at $336 \mathrm{~Hz}$ are discussed and compared with an analysis of the flame response at $176 \mathrm{~Hz}$, which is detailed in another paper [31].

\section{Flame transfer function}

The flame transfer function is defined as the ratio of the relative heat release rate fluctuations from the flame over the relative velocity fluctuations of the flow in the frequency domain $(f)$ :

$$
F(f)=\frac{\dot{Q}^{\prime}(f) / \bar{Q}}{u^{\prime}(f) / \bar{u}}
$$


where $\overline{(\cdot)}$ denotes the mean value of a quantity, and $(\cdot)^{\prime}$ denotes its fluctuation. The FTF can also be expressed in terms of a gain $G$ and a phase $\Phi$ :

$$
F(f)=G(f) \mathrm{e}^{\mathrm{i} \Phi(f)},
$$

The gain and the phase of the FTF for propane flames with $S_{L}$ varying from 0.2 to $0.32 \mathrm{~m} / \mathrm{s}$ are presented in Fig. 3 .

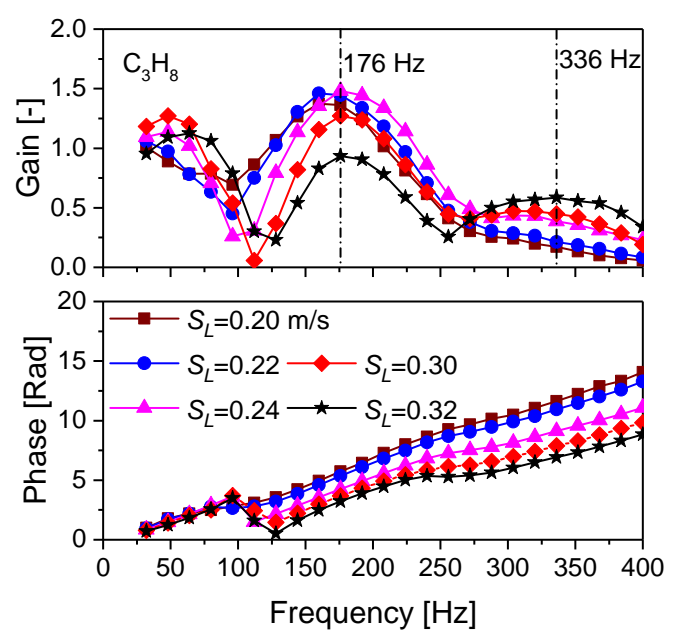

FIGURE 3. FLAME TRANSFER FUNCTIONS FOR PROPANE FLAMES WITH A FORCING AMPLITUDE OF 10\%, FOR DIFFERENT VALUES OF $S_{L}(\mathrm{~m} / \mathrm{s})$.

They feature the typical trends of swirl stabilized flames. At low frequencies, the gain approaches one and the phase approaches zero [36]. The gain presents three local maxima at $64 \mathrm{~Hz}, 176 \mathrm{~Hz}, 336 \mathrm{~Hz}$, and two local minima at $112 \mathrm{~Hz}$ and $256 \mathrm{~Hz}$. This trend is the result of the interaction between the fluctuations of the base of the flame and the oscillations of the top region of the flame due to FVR, as described in the introduction section. The phase increases linearly with the forcing frequency showing a jump to lower values at around $112 \mathrm{~Hz}$. Increasing the laminar burning velocity, no shift in the frequency of the local extrema is observed. However, when $S_{L}$ increases, the gain at $64 \mathrm{~Hz}$ and $176 \mathrm{~Hz}$ increases first, and then it decreases. In contrast, at $336 \mathrm{~Hz}$, the gain monotonically increases with increasing laminar burning velocity. No clear trend of the gain at $112 \mathrm{~Hz}$ and $256 \mathrm{~Hz}$ is observed changing $S_{L}$. The phase, at frequencies lower than $112 \mathrm{~Hz}$, is not affected by the change in laminar burning velocity, while for higher frequencies it decreases with $S_{L}$. Similar results are obtained for the methane flames. The effect of the laminar burning velocity and equivalence ratio on the gain response at $336 \mathrm{~Hz}$ are presented in Fig. 4. It shown that by in- creasing $\phi$, and consequently $S_{L}$, the gain at $336 \mathrm{~Hz}$ increases linearly for both fuels.

\section{Dynamics of the flame}

To better understand the response of the flame to acoustic perturbation of the flow at $336 \mathrm{~Hz}$, the phase-locked Abeldeconvoluted $\mathrm{OH}^{*}$ chemiluminescence images are reported in Fig. 5 for propane and in Fig. 6 for methane.

These images are normalized with respect to the maximum value of $\mathrm{OH}^{*}$ chemiluminescence intensity considering all the flames. This normalizing value is kept constant for all the experimental conditions. A 0.4 iso-contour (in black) is superimposed to the images to ease the visualization of the flame motion. A previous study from Di Sabatino etal. [9] shows that different values of the iso-contour do not affect the analysis of the results. Since the flame vortex roll-up at the tip of the flame is an important mechanism controlling the response of the flame to acoustic forcing $[6,15,34,37-40]$, the ellipse in white is used to highlight its maximum size during the forcing cycle. Changing fuel and equivalence ratio, the maximum size of the ellipse changes. This is discussed later in this paper. Moreover, a black straight solid line in Fig. 5 and Fig. 6 divides each image into two regions. A top region, above the line, that is positioned to include all the effects of the flame vortex roll-up on the response of the flame to acoustic forcing, and a bottom region below the line. The $\mathrm{OH}^{*}$ chemiluminescence intensity integrated over each region during the forcing period is reported in Fig. 7. These signals are normalized with their average value in the same region of the flame over the whole forcing cycle.

For both fuels, the relative amplitude of the fluctuations of the bottom region with respect to the top region changes with $S_{L}$. More precisely, the amplitude of the fluctuations of both regions increases with increasing the laminar burning velocity. Moreover, the phase difference between two regions changes with $S_{L}$. The fluctuations of the two regions are almost out-of-phase for the lower values of $S_{L}$, while they are almost in-phase for the higher values of laminar burning velocity. This trend is analyzed in the Discussion section.

\section{Velocity fields}

The velocity fields of the unforced flames are presented in Fig. 8. Two equivalence ratios for each fuel are reported as examples.

It is possible to observe the presence of an inner recirculation zone (IRZ) surrounded by an annular jet (AJ) enclosed by an outer recirculation zone (ORZ) that are typical of swirl flows. A 0.5 iso-contour of the Q-criterion is superimposed to the flow field to highlight the ORZ. For each fuel, it is shown that the outer recirculation zone reduces its size and it moves upstream towards the burner plate, when $S_{L}$ is increased. Considering the propane flames flow field (right side of Fig. 8), the perimeter 

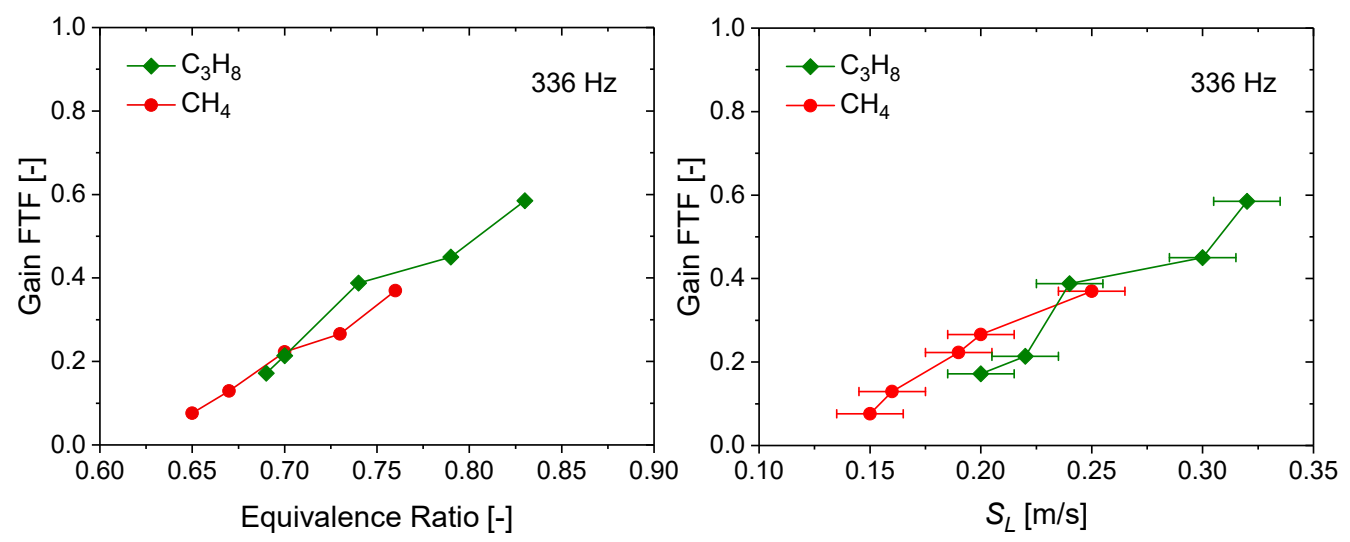

FIGURE 4. GAIN RESPONSE AT $336 \mathrm{~Hz}$ AS A FUNCTION OF THE EQUIVALENCE RATIO (LEFT) AND LAMINAR BURNING VELOCITY (RIGHT). THE ERROR BARS REPRESENT THE DISCREPANCY IN THE VALUE OF $S_{L}$.

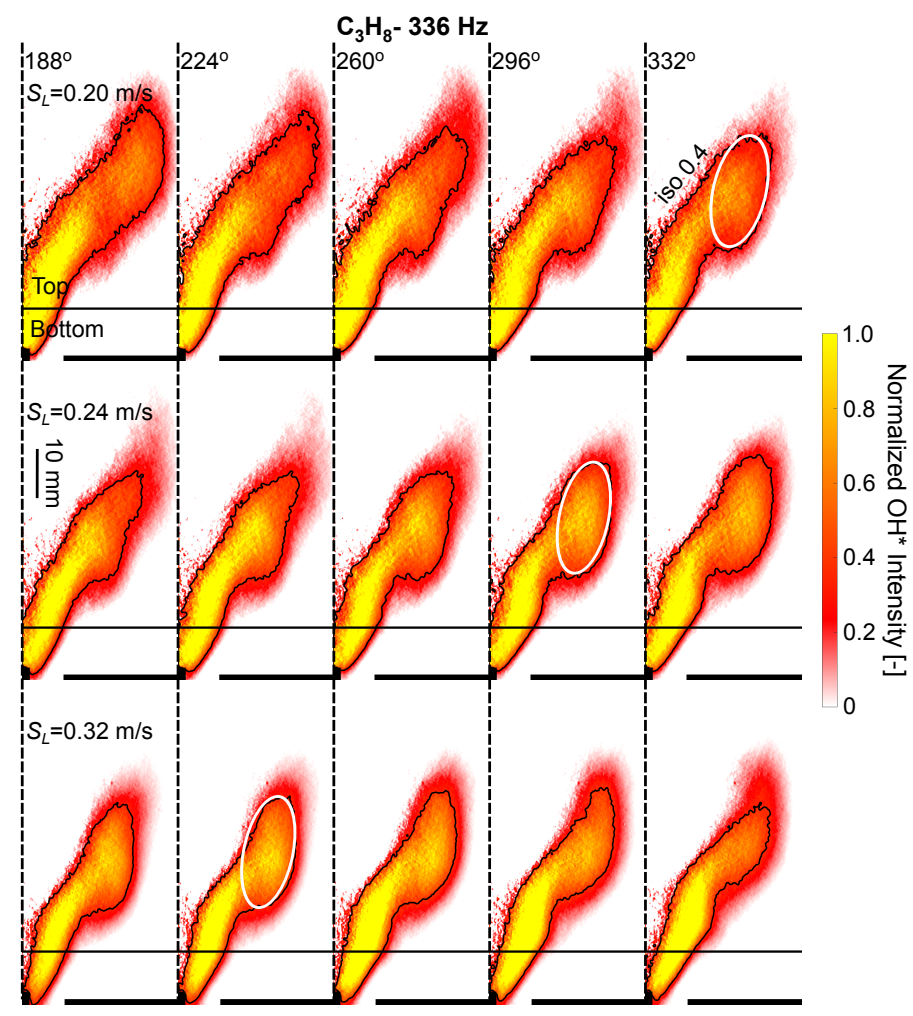

FIGURE 5. PHASE-LOCKED ABEL-DECONVOLUTED OH* CHEMILUMINESCENCE IMAGES OF PROPANE FLAMES FORCED AT $336 \mathrm{~Hz}$.

of the ORZ decreases from around $45 \mathrm{~mm}$ for $S_{L}=0.22 \mathrm{~mm}$ to $35 \mathrm{~mm}$ for $S_{L}=0.32 \mathrm{~mm}$. Whereas, the distance of center mass of the ORZ from the combustor dump plate decreases from $27 \mathrm{~mm}$ to $22 \mathrm{~mm}$. A similar trend is observed for methane. This behavior is discussed in the following section.

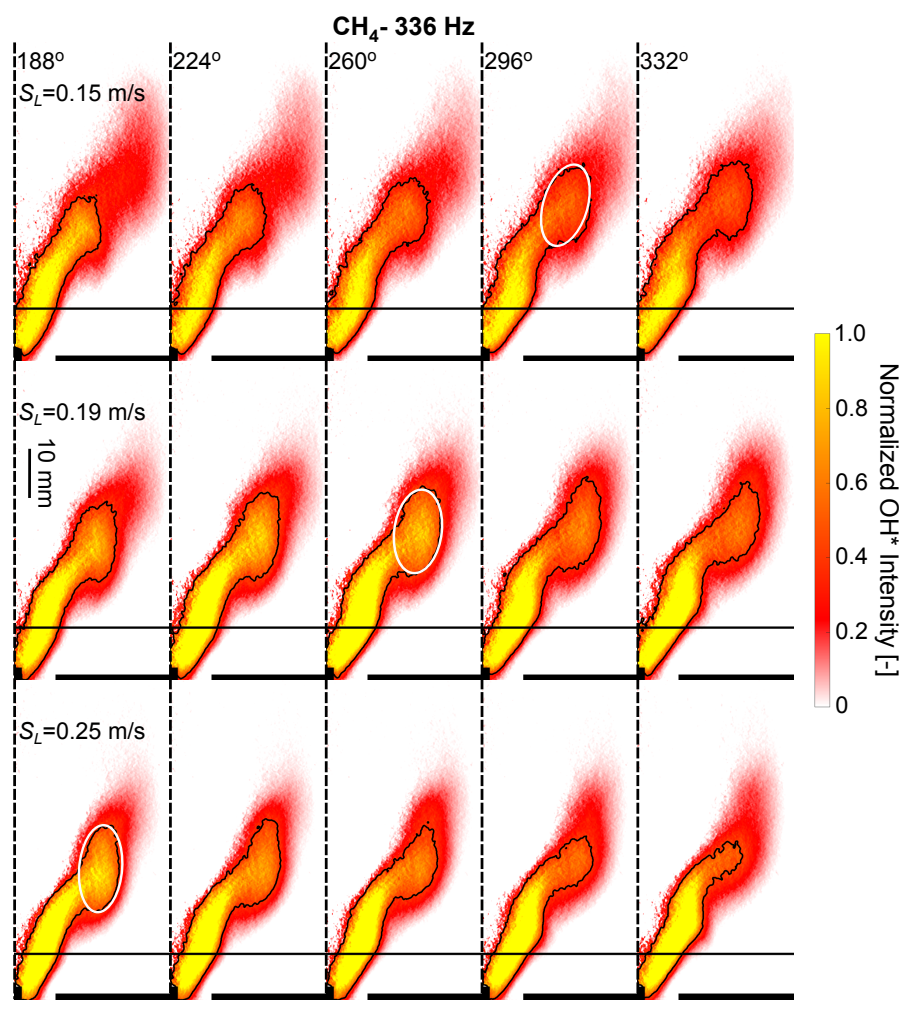

FIGURE 6. PHASE-LOCKED ABEL-DECONVOLUTED OH* CHEMILUMINESCENCE IMAGES OF METHANE FLAMES FORCED AT $336 \mathrm{~Hz}$.

\section{Discussion}

As shown in [34,40], the magnitude of the response of a premixed swirl flame to acoustic forcing, i.e. the gain of the FTF, is mainly controlled by the interaction between the fluctuations of the bottom and top region of the flame. The oscillations of the bottom region are driven by the fluctuations of the flame base 

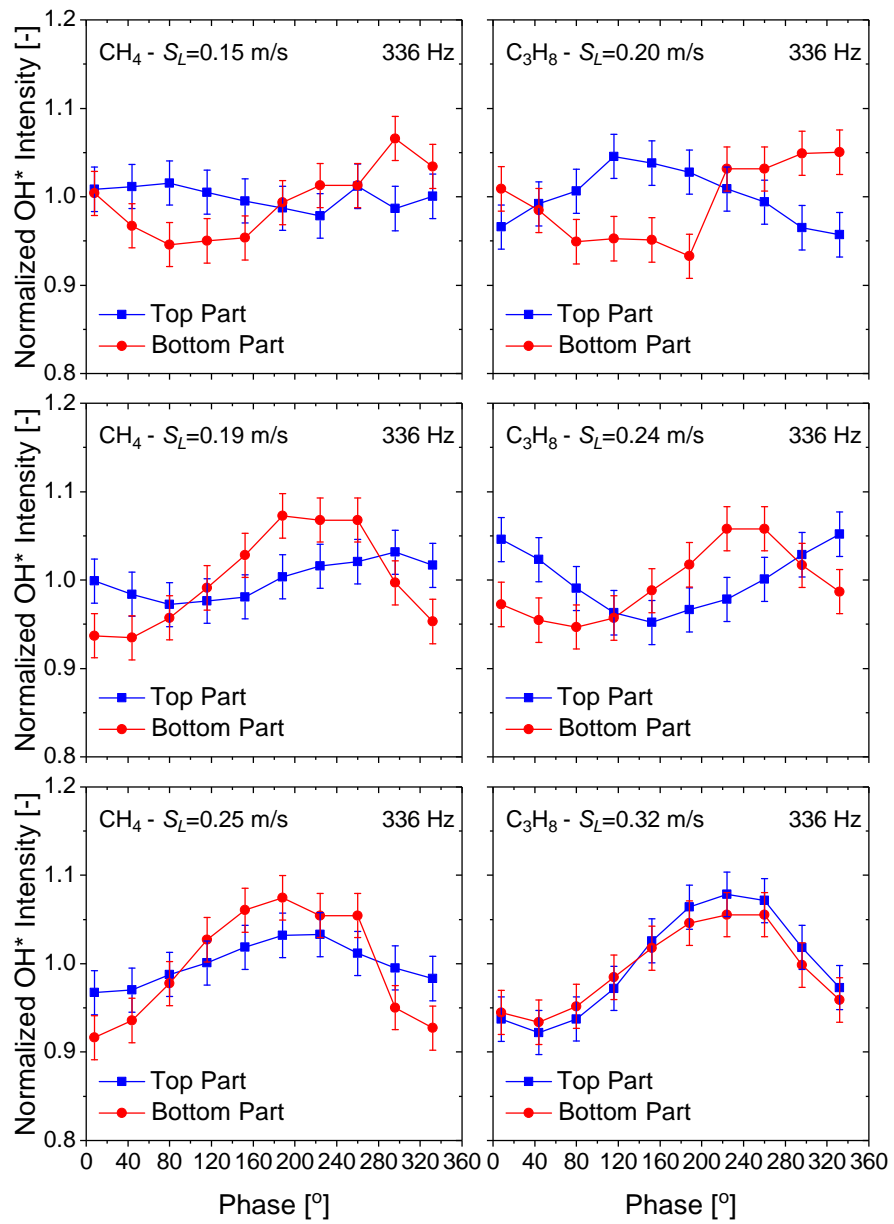

FIGURE 7. EVOLUTION OF THE NORMALIZED OH* CHEMILUMINESCENCE OF METHANE (LEFT) AND PROPANE (RIGHT) FLAMES WITH $10 \%$ FORCING AMPLITUDE IN THE TOP AND BOTTOM REGIONS. THE ERROR BARS REPRESENT THE UNCERTAINTY IN THE DIVISION BETWEEN TOP AND BOTTOM REGIONS.

angle, generated by the swirl number oscillations, while the fluctuations of the top region are controlled by the flame vortex rollup. From Fig. 7, it is possible to observe that both regions of the flame are oscillating during the forcing cycle highlighting that both regions are contributing to the response of the flame to acoustic forcing at $336 \mathrm{~Hz}$. From Figs. 5 and 6, the maximum perimeter of the flame vortex roll-up $\left(\mathrm{FVR}_{\max }\right)$ can be estimated as the perimeter of the white ellipse that best fits in the 0.4 isocontour. The value of the gain of the FTF at $336 \mathrm{~Hz}$ as a function of the FVR $\mathrm{Fax}_{\text {ax }}$ is reported in Fig. 9.

A linear behavior can be observed, highlighting that the FVR is an important parameter determining the flame response to acoustic forcing at $336 \mathrm{~Hz}$. On the other hand, it is important to note that a non-zero value of $\mathrm{FVR}_{\max }$ results in a zero value of the gain. If the motion of the bottom region was negligible compared to the top region, a gain equal to zero should result from a zero value of the $\mathrm{FVR}_{\max }$. In other words, the gain is zero when the $\mathrm{FVR}_{\max }$ is not zero because the motion of the bottom region of the flame is destructively interfering with the fluctuation of the top region reducing the global response of the flame to acoustic forcing. An additional explanation for this non-zero offset could be that an ellipse of finite perimeter could fit in the thickness of the turbulent flame brush even if the FVR is not present (see Fig. 2). This is because the thickness of the turbulent flame brush is of the same order as the size of the ellipse (see Figs. 5 and 6).

The maximum perimeter of the flame vortex roll-up is plotted as a function of $S_{L}$ in Fig. 10.

It is possible to observe a linear trend between the laminar burning velocity and the FVR $\max$ for $S_{L}<0.23 \mathrm{~m} / \mathrm{s}$. This suggests that, below this threshold value, a higher value of $S_{L}$ increases the ability of the flame to adapt to sudden changes in the flow field generated by the impinging vortex producing a bigger flame vortex roll-up. For $S_{L}>0.23 \mathrm{~m} / \mathrm{s}$ and for each fuel, the trend between $S_{L}$ and the FVR $\mathrm{Fax}_{\max }$ diverges from the linearity, suggesting that other parameters are also affecting the maximum perimeter of the FVR. From Fig. 8, it is possible to observe that for high values of $\phi$, i.e. high values of $S_{L}$, the velocity field changes. In particular, the ORZ becomes smaller, and it moves toward the combustor dump plate. It is reasonable to assume that the strength of the acoustically generated vortex is affected similarly. To explain this behavior, it is also reasonable to suppose that the flames that features higher values of laminar burning velocity stabilize closer to the nozzle tip, and consequently closer to the shear layer where the AGV is shed.

The vertical distance $(d)$ between the flame front and the nozzle tip (see Fig. 2) as a function of $S_{L}$ is reported in Fig. 11. This distance is evaluated from the $\mathrm{OH}^{*}$ chemiluminescence images of the unforced flames. From the same images, the flame base angle is evaluated, and it is important to underline that it is constant, $35 \pm 2^{\circ}$ from the burner axis, for all the conditions investigated. For both fuels, the stabilization distance decreases rapidly for higher values of laminar burning velocity. However, propane flames have higher values of $S_{L}$ than methane, but larger $d$ are measured. This difference in the stabilization of swirl flames will be investigated in future studies. It has been suggested in $[8,41]$, that the closer the flame is to the shear layer, the higher is the temperature rise in that region, and the weaker is the acoustically generated vortex. As proposed in [8, 42-44] there are several phenomena that can weaken a vortex: the gas expansion, the baroclinic generation of vorticity, and the viscous diffusion. All these mechanisms are enhanced by the increase in the temperature of the gas due to closer proximity of the flame. Considering the flames analyzed in this study, when the equivalence ratio is increased beyond a particular value, it is reasonable to assume that the strength of the AGV starts to decrease due to the mechanisms explained above. Therefore, the strength of the 

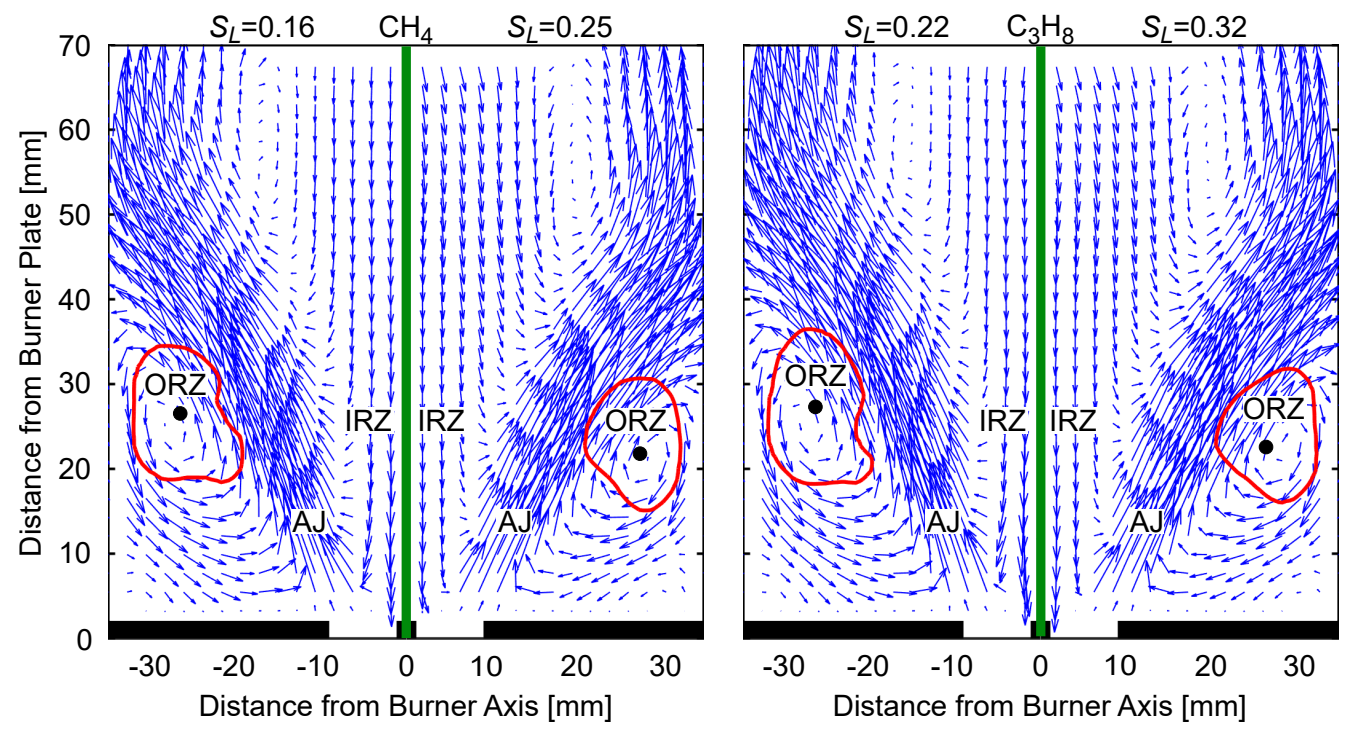

FIGURE 8. UNFORCED VELOCITY FIELDS OF METHANE (LEFT) AND PROPANE (RIGHT) FLAMES. THE CENTER OF MASS OF THE ORZs IS HIGHLIGHTED BY A SOLID BLACK DOT.

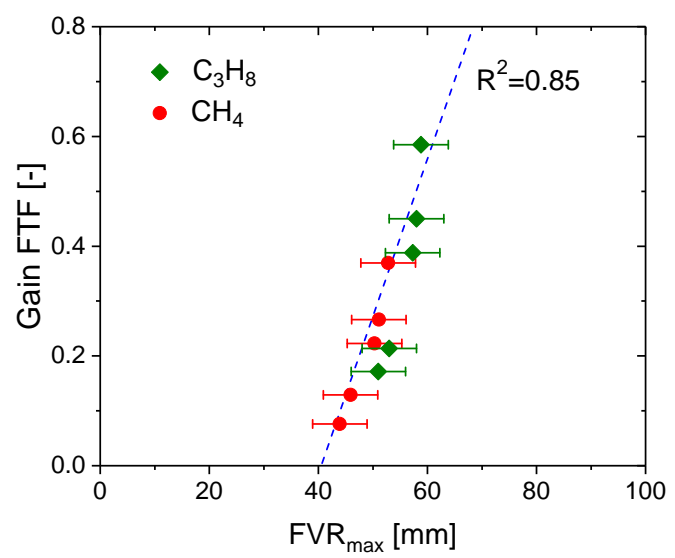

FIGURE 9. GAIN OF THE FTF AT $336 \mathrm{~Hz}$ AS A FUNCTION OF FVR $\max$ FOR ALL THE CONDITIONS ANALYZED IN THIS STUDY. THE ERROR BARS REPRESENT THE UNCERTAINTY IN THE FITTING PROCESS OF THE WHITE ELLIPSE INTO THE ISOCONTOUR OF NORMALIZED OH* CHEMILUMINESCENCE.

flame vortex roll-up is also affected, and consequently the gain of the FTF at $336 \mathrm{~Hz}$.

An increase in equivalence ratio results in both an increase in $S_{L}$ and in flame temperature, since only lean flames are considered here. This increase in flame temperature can also enhance the gas expansion and the baroclinic generation of vorticity and, consequently, weaken the AGV. In Fig. 12, the laminar burning velocity is plotted as a function of the ratio of the adiabatic flame temperature, $T_{a d}$, to the temperature of the unburnt gases, $T_{u}=300 \mathrm{~K}$. Of course, it would have been more accurate

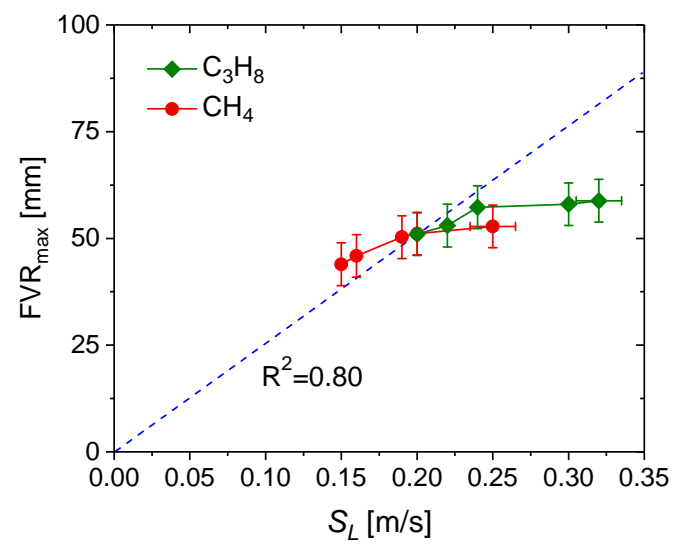

FIGURE 10. THE FVR max $_{\text {ax }}$ AT $336 \mathrm{~Hz}$ AS A FUNCTION OF THE LAMINAR BURNING VELOCITY FOR ALL THE EXPERIMENTAL CONDITIONS ANALYZED IN THIS STUDY. THE VERTICAL ERROR BARS REPRESENT THE UNCERTAINTY IN DETERMIN-

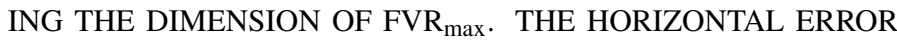
BARS REPRESENT THE DISCREPANCY IN THE VALUE OF $S_{L}$. THE DASHED BLUE LINE IS INTERPOLATED CONSIDERING ONLY THE THREE LOWEST $S_{L}$ OF EACH FUEL.

to measure the temperatures of the burnt and fresh gases. In any case, this coarse analysis shows that $S_{L}$ and $T_{a d} / T_{u}$ are closely related, so they can both contribute in weakening the AGV. It is also interesting to notice that $S_{L}$ has a broader range of variation, more than a factor of 2 , than $T_{a d} / T_{u}$, with about $25 \%$. This suggest that the effect of the increase in flame temperature is perhaps secondary compared to the effect of the increase in laminar burn- 


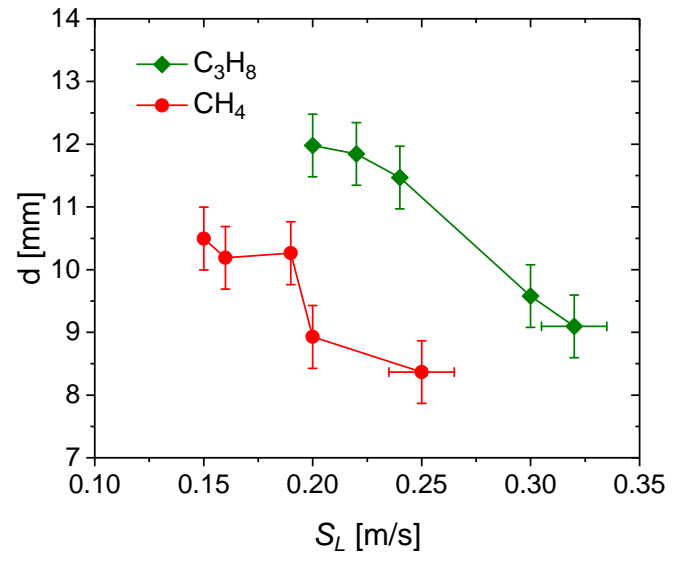

FIGURE 11. STABILIZATION DISTANCE OF THE UNFORCED FLAMES AS A FUNCTION OF THE LAMINAR BURNING VELOCITY. THE VERTICAL ERROR BAR REPRESENTS THE UNCERTAINTY IN THE POSITION OF THE FLAME FRONT. THE HORIZONTAL ERROR BAR REPRESENTS THE DISCREPANCY IN THE VALUE OF $S_{L}$ FROM LITERATURE AND SIMULATIONS.

ing velocity. To further assess the relative impact of each of these two effects on the AGV, it would be necessary to decouple them.

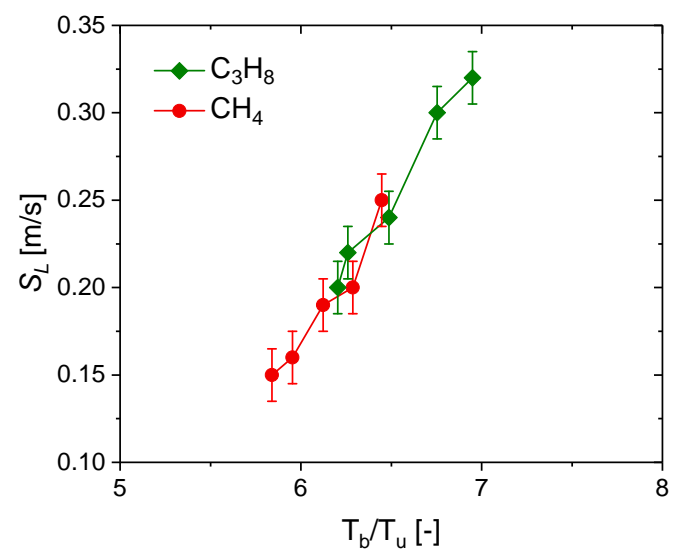

FIGURE 12. LAMINAR BURNING VELOCITY AS A FUNCTION OF $T_{a d} / T_{u}$. THE ERROR BARS REPRESENT THE DISCREPANCY IN THE VALUE OF $S_{L}$.

Finally, Fig. 3 shows that at $176 \mathrm{~Hz}$ the response of the flame to acoustic forcing is also very sensitive to changes in the burning velocity. At this frequency, the gain of the FTF shows a nonmonotonic trend with increasing $S_{L}$. A detailed description and discussion of the results obtained at $176 \mathrm{~Hz}$ can be found in [31]. In the present study, a brief summary of the main findings is given, in order to broaden the discussion conducted for $336 \mathrm{~Hz}$.
At $176 \mathrm{~Hz}$, the dynamics of the bottom region of the flame is negligible compared to the top one, which is controlled by the flame vortex roll-up. For the ten flames considered, the FVR is then the dominant mechanism controlling the response of the flame at $176 \mathrm{~Hz}$. At this frequency, the effect of $S_{L}$ on the FVR is similar to what is observed at $336 \mathrm{~Hz}$. The capability of the flame to wrap around the AGV is enhanced with increasing $S_{L}$, but if $S_{L}$ becomes too large, the distance between the flame base and the nozzle tip reduces, resulting in a weaker AGV. Therefore, even if the trend of the gain is not the same at 176 and $336 \mathrm{~Hz}$, the conclusions regarding the effect of $S_{L}$ on the FVR hold for both maximums.

\section{CONCLUSIONS}

The influence of the laminar burning velocity on the transfer function of swirl flames has been investigated. Five different equivalence ratios for both methane and propane fuels have been investigated. The phase-locked images of $\mathrm{OH}^{*}$ chemiluminescence have been used to characterize the flame response to acoustic forcing in terms of flame vortex roll-up. The effect of $S_{L}$ on the velocity field has also been analyzed using PIV. The main findings of this study are:

1. For each fuel, modifying $S_{L}$ affects mainly the gain at its local maximums, i.e. at $176 \mathrm{~Hz}$ and $336 \mathrm{~Hz}$. Increasing $\phi$, i.e. increasing $S_{L}$, the gain response at $336 \mathrm{~Hz}$ increases approximately linearly.

2. The flame vortex roll-up has a strong impact on the gain at $336 \mathrm{~Hz}$. However, the fluctuations of the bottom region of the flame are still active at this frequency and cannot be neglected.

3. The maximum size of the FVR scales linearly with the laminar burning velocity, for values of $S_{L}$ lower than a specific value $(0.23 \mathrm{~m} / \mathrm{s}$ in this study). On the other hand, the trend of the $\mathrm{FVR}_{\max }$ diverges from linearity, for values of $S_{L}$ higher than this threshold. This could be due to the decrease in the distance between the flame front and the shear layer region that weakens the acoustically generated vortex.

4. The increase in equivalence ratio also results in an increase in flame temperature that can also weaken the AGV. Additional investigations will be necessary to assess the relative impact of the flame temperature and laminar burning velocity on the stabilization distance and the strength of the AGV.

Finally, to conclude on a more practical note, this study shows that for frequencies at which the flame is the most responsive to acoustic perturbations, i.e., at the maximums of the gain, for a given fuel, an increase in $S_{L}$ generally increases the magnitude of the response, but not always. If the stabilization distance from the injection nozzle is not changed by $S_{L}$, then the magnitude of the gain increases. However, if the increase in $S_{L}$ and $T_{a d}$ 
is strong enough to decrease the stabilization distance, the properties of the AGV are changed. In this case, depending on the frequency and the mechanisms controlling the flame dynamics, the gain can increase (as at $336 \mathrm{~Hz}$ in this study), or decrease (as at 64 and $176 \mathrm{~Hz}$ ). This information is valuable for engine designers as it allows them to anticipate changes in the flame response to acoustic perturbations and, thus, thermoacoustic interactions, when the fuel (composition) is varied.

\section{ACKNOWLEDGMENT}

This work is funded by the King Abdullah University of Science and Technology and by the Deutsche Forschungsgemeinschaft through the GECCO project.

\section{REFERENCES}

[1] Candel, S., 2002. "Combustion dynamics and control: Progress and challenges". Proc. Combust. Inst., 29(1), pp. 1-28.

[2] Lieuwen, T. C., and Yang, V., eds., 2005. Combustion Instabilities in Gas Turbine Engines, Vol. 210 of Progress in Astronautics and Aeronautics. AIAA, Inc.

[3] Ducruix, S., Schuller, T., Durox, D., and Candel, S., 2003. "Combustion dynamics and instabilities: Elementary coupling and driving mechanisms". J. Propul. Power, 19(5), pp. 722-734.

[4] Palies, P., Durox, D., Schuller, T., and Candel, S., 2011. "Nonlinear combustion instability analysis based on the flame describing function applied to turbulent premixed swirling flames". Combust. Flame, 158, pp. 1980-1991.

[5] Schuller, T., Durox, D., and Candel, S., 2003. "A unified model for the prediction of laminar flame transfer functions: comparisons between conical and V-flame dynamics". Combust. Flame, 134(1-2), pp. 21-34.

[6] Bellows, B., Bobba, M., Forte, A., Seltzman, J., and Lieuwen, T., 2007. "Flame transfer function saturation mechanisms in a swirl-stabilized combustor". Proc. Combust. Inst., 31(2), pp. 3181-3188.

[7] Kim, K., Lee, J., Quay, B., and Santavicca, D., 2010. "Spatially distributed flame transfer functions for predicting combustion dynamics in lean premixed gas turbine combustors". Combust. Flame, 157(9), pp. 1718-1730.

[8] Bunce, N., Quay, B., and Santavicca, D., 2014. "Interaction between swirl number fluctuations and vortex shedding in a single-nozzle turbulent swirling fully-premixed flame combustor". J. Eng. Gas Turbines Power, 136, pp. 021503-111.

[9] Di Sabatino, F., Guiberti, T. F., Boyette, W. R., Roberts, W. L., Moeck, J. P., and Lacoste, D. A., 2018. "Effect of pressure on the transfer functions of premixed methane and propane swirl flames". Combust. Flame, 193, pp. 272-282.
[10] Bellows, B., Neumeier, Y., and Lieuwen, T., 2006. "Forced response of a swirling, premixed flame to flow disturbances”. J. Propul. Power, 22(5), pp. 1075-1084.

[11] Kim, K., Lee, J., Quay, B., Santavicca, D., Kim, K., and Srinivasan, S., 2008. "Effect of flame structure on the flame transfer function in a premixed gas turbine combustor". In Proc. ASME Turbo Expo. paper no. GT2008-51014.

[12] Freitag, E., Konle, H., Lauer, M., Hirsch, C., and Sattelmayer, T., 2006. "Pressure influence on the flame transfer function of a premixed swirling flame". In Proc. ASME Turbo Expo. paper no. GT2006-90540.

[13] Bunce, N., Lee, J., Quay, B., and Santavicca, D., 2011. "Mixture-forced flame transfer function measurement and mechanism in a single-nozzle combustor at elevated pressure”. In Proc. ASME Turbo Expo. paper no. GT201146744.

[14] Hochgreb, S., Dennis, D., Ayranci, I., Bainbridge, W., and Cant, S., 2013. "Forced and self-excited instabilities from lean premixed, liquid-fuelled aeroengine injectors at high pressure and temperatures". In Proc. ASME Turbo Expo. paper no. GT2013-95311.

[15] Oberleithner, K., Schimek, S., and Paschereit, C., 2015. "Shear flow instabilities in swirl-stabilized combustor and their impact on the amplitude dependent flame response: A linear stability analysis". Combust. Flame, 162, pp. 86-99.

[16] Gatti, M., Gaudron, R., Mirat, C., Zimmer, L., and Schuller, T., 2018. "A comparison of the transfer functions and flow fields of flames with increasing swirl number". In Proc. ASME Turbo Expo. paper no. GT2018-76105.

[17] Gatti, M., Gaudron, R., Mirat, C., Zimmer, L., and Schuller, T., 2018. "Impact of swirl and bluff-body on the transfer function of premixed flames". Proc. Combust. Inst., Article in Press.

[18] Gaudron, R., Gatti, M., Mirat, C., and Schuller, T., 2017. "Impact of the injector size on the transfer functions of premixed laminar conical flames”. Combust. Flame, 179, pp. 138-153.

[19] Lacoste, D., Moeck, J., Durox, D., Laux, C., and Schuller, T., 2013. "Effect of nanosecond repetitively pulsed discharges on the dynamics of a swirl-stabilized lean premixed flame". J. Eng. Gas Turbines Power, 135, p. 101501.

[20] Gupta, A., Lilley, D., and Syred, N., 1984. Swirl Flows. Abacus Press, Tunbridge Wells, England.

[21] Di Sabatino, F., Lacoste, D. A., and Roberts, W. L., 2017. "A detailed characterization of a high pressure experimental apparatus for flame dynamics studies". 10th U.S. National Combustion Meeting, p. Paper no. 2H03.

[22] Goodwin, D., Moffat, H., and Speth, R., 2009. "Cantera: An object-oriented software toolkit for chemical kinetics, thermodynamics, and transport processes". Caltech, Pasadena, CA.

[23] Wang, H., You, X., Joshi, A., Davis, S., Laskin, A., Egol- 
fopoulos, F., and Law, C., 2007. "USC mech version II". High-Temperature Combustion Reaction Model of H2/CO/C1-C4 Compounds.

[24] Metghalchi, M., and Keck, J. C., 1980. "Laminar burning velocity of propane-air mixtures at high temperature and pressure". Combust. Flame, 38, pp. 143-154.

[25] Yamaoka, I., and Tsuji, H., 1984. "Determination of burning velocity using counterflow flames". Symp. (Int.) Combust., pp. 1883-1892.

[26] Yu, G., Law, C. K., and Wu, C. K., 1986. "Laminar flame speed of hydrocarbon + air mixtures with hydrogen addition". Combust. Flame, 63, pp. 339-347.

[27] Davis, S. G., and Law, C. K., 1998. "Determination of and fuel structure effects on laminar flame speeds of $\mathrm{C}_{1}$ to $\mathrm{C}_{8}$ hydrocarbons". Combust. Sci. and Tech., 140, pp. 427449.

[28] Zhao, Z., Kazakov, A., Li, J., and Dryer, F. L., 2004. "The initial temperature and $\mathrm{N}_{2}$ dilution effect on the laminar flame speed of propane/air". Combust. Sci. and Tech., 176, pp. 1705-1723.

[29] Mazas, A. N., Fiorina, B., Lacoste, D. A., and Schuller, T., 2011. "Effect of water vapor addition on the laminar burning velocity of oxygen-enriched methane flames". Combust. Flame, 158, pp. 2428-2440.

[30] Preetham, Thumuluru, S., Santosh, H., and Lieuwen, T., 2010. "Linear response of laminar premixed flames to flow oscillations: unsteady stretch effects". J. Propul. Power, 26(3), pp. 524-532.

[31] Di Sabatino, F., Guiberti, T. F., Moeck, J. P., Roberts, W. L., and Lacoste, D. A., 2018. "Effects of fuel and equivalence ratio on the transfer functions of methane and propane premixed swirl flames". J. Propul. Power, Submitted.

[32] Hurle, I., Price, R., Sugden, T., and Thomas, A., 1968. "Sound emission from open turbulent premixed flames". , Ser. A, 303(1475), pp. 409-427.

[33] Price, R., Hurle, I., and Sugden, T., 1971. "Optical studies of the generation of noise in turbulent flames". pp. 10931102.

[34] Palies, P., Durox, D., Schuller, T., and Candel, S., 2011. "Experimental study on the effect of swirler geometry and swirl number on flame describing functions". Combust. Sci. and Tech., 183, pp. 704-717.

[35] Jeong, J., and Hussain, F., 1995. "On the identification of a vortex”. J. Fluid Mech., 285, pp. 69-94.

[36] Polifke, W., and Lawn, C., 2007. "On the low-frequency limit of flame transfer functions". Combust. Flame, 151(3), pp. 437-451.

[37] Külsheimer, C., and Büchenr, H., 2002. "Combustion dynamics of turbulent swirling flames". Combust. Flame, 131, pp. 70-84.

[38] Kang, D., Culik, F., and Ratner, A., 2007. "Combustion dynamics of a low-swirl combustor". Combust. Flame, 151, pp. 412-425.

[39] Huang, Y., and Yang, V., 2009. "Dynamics and stability of lean-premixed swirl-stabilized combustor”. Prog. Energy Combust. Sci., 35 (4), pp. 293-364.

[40] Palies, P., Durox, D., Schuller, T., and Candel, S., 2010. "The combined dynamics of swirler and turbulent premixed swirling flames". Combust. Flame, 157, pp. 1698-1717.

[41] Palies, P., Schuller, T., Durox, D., Gicquel, L. Y. M., and Candel, S., 2011. "Acoustically perturbed turbulent premixed swirling flames". Phys. Fluids, 23, pp. 037101-1037101-15.

[42] Coats, C., 1994. "Coherent structures in combustion". Prog. Energy Combust. Sci., 22 (5), pp. 427-509.

[43] Renard, P. H., Thevenin, D., Rolon, J. C., and Candel, S., 2000. "Dynamic of flame/vortex interaction". Prog. Energy Combust. Sci., 26, pp. 225-282.

[44] Emerson, B., O’Connor, J., Juniper, M., and Lieuwen, T., 2012. "Density ratio effects on the reacting bluff-body flow field characteristics". J. Fluid Mech., 706, pp. 219-250. 